\title{
MESSAGE FROM EDITORIAL DESK
}

Chitwan Medical College takes pride to be the first Medical College in Nepal to be awarded ISO 9001:2008 certification for its Quality Management System. It's an added responsibility on our part to deliver quality education and health services to our clients continuously and consistently in days to come. The 6th issue of Journal of Chitwan Medical College is one of the proofs of our dedication and commitment to maintain the standard.

I'd like to take this opportunity to thank all who are involved in this process, beginning with the authors who submit their papers, the reviewers, by lending their expertise and time, giving their opinions on technical, scientific, statistical and experimental analysis, as well as state of the art and those who helped during the page setting, printing and publishing.

JCMC is currently accepting manuscripts of articles for the upcoming issue. The articles should be based on original qualitative or quantitative research, an innovative conceptual framework, or a substantial literature review that opens new areas of inquiry and investigation. Case studies and analytical articles are also welcome. The journal recognizes that the articles and research papers of allied health sciences also are of great importance and feel that they too should be published for the benefit of the readers. So we invite and also encourage researchers, students and practioners to submit such articles of whatever the field of health science they may be or belong to.

The reviewer's comments are useful by helping the Editorial Board make decisions regarding acceptance of a received paper; providing criticisms that help the authors to improve their papers as well as to maintain the quality and pertinence of JCMC. I request the authors to take as many steps as possible to make their articles right before it is submitted. A worrying problem is poor English, especially when the scientific work is novel and deserves publication.

Please direct your manuscripts, questions, or comments to jcmc@cmc.edu.np. I hope you will enjoy reading this edition of JCMC and be benefitted by it.

I wish you all the happiest New Year 2014.

With warm regards,

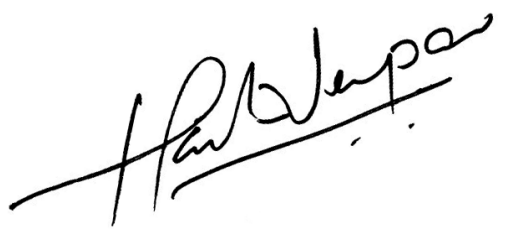

Prof Dr Harish Chandra Neupane

Editor in Chief 